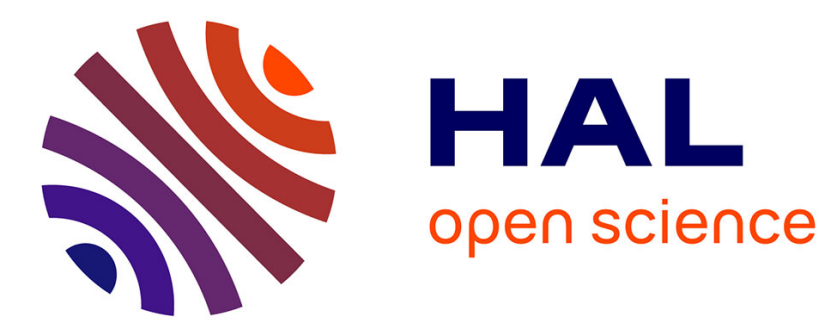

\title{
Verbatim and gist memory in aging
}

Marlène Abadie, Elisa Gavard, Fabrice Guillaume

\section{To cite this version:}

Marlène Abadie, Elisa Gavard, Fabrice Guillaume. Verbatim and gist memory in aging. Psychology and Aging, In press, 10.1037/pag0000635 . hal-03315760

\section{HAL Id: hal-03315760 \\ https://hal.science/hal-03315760}

Submitted on 5 Aug 2021

HAL is a multi-disciplinary open access archive for the deposit and dissemination of scientific research documents, whether they are published or not. The documents may come from teaching and research institutions in France or abroad, or from public or private research centers.
L'archive ouverte pluridisciplinaire HAL, est destinée au dépôt et à la diffusion de documents scientifiques de niveau recherche, publiés ou non, émanant des établissements d'enseignement et de recherche français ou étrangers, des laboratoires publics ou privés. 
Running head: Verbatim and gist memory in aging

(C) 2021, American Psychological Association. This paper is not the copy of record and may not exactly replicate the final, authoritative version of the article. Please do not copy or cite without authors' permission. The final article will be available, upon publication, via its DOI: 10.1037/pag0000635

\section{Verbatim and gist memory in aging}

Marlène Abadie, Elisa Gavard \& Fabrice Guillaume

Laboratoire de Psychologie Cognitive, Aix-Marseille Université, France.

\section{Author Note}

This work was supported by a grant from the French National Research Agency (No. ANR19-CE28-0017-01) to Marlène Abadie. The funding source was not involved in the conduct of the research nor in the preparation of the article. The authors declare that there are no conflicts of interest. The study was presented at the $2^{\text {nd }}$ edition of the Recollection, familiarity, and novelty detection conference in Liège on October 3-4, 2019, and at the Journées d'Etude du Vieillissement in Lyon on May 20-21, 2021. Data and material for this study, as well as additional analyses are openly available at the Open Science Framework (OSF), https://osf.io/7tzgw/?view_only=5ed54adada5040f8bb47cf7444e4f39f. We thank Roxane Consoloni and Marine Vialla for her assistance with data collection. Correspondence should be addressed to Marlène Abadie, Laboratoire de Psychologie Cognitive, CNRS \& AMU, UMR7290, Bâtiment 9 Case D, 3, place Victor Hugo, 13331 Marseille Cedex 3, France. marlene.abadie@univ-amu.fr, phone: +33(0) 413550974. 


\begin{abstract}
The ability to remember episodic details of prior events declines with normal aging. The present study aimed at determining whether these declines are restricted to verbatim traces of items per se or extend to gist traces of their meaning. Younger $(n=63)$ and older adults $(\mathrm{n}=46)$ studied a list including related (strong gist activation) and unrelated words (weak gist activation) and performed a recognition test consisting of targets, related distractors, and unrelated distractors. Gist memory increased in the strong relative to the weak gist condition in both age groups. Whereas both younger and older adults could retrieve gist traces of target words, older adults were impaired in their ability to retrieve their verbatim traces resulting in increased false recognition of related distractors. These findings suggest an age-related decrease in the ability to retrieve verbatim details of past episodes accompanied by an increase in reliance on gist memory.
\end{abstract}

\title{
Keywords:
}

aging, gist memory, verbatim memory, conjoint recognition, multinomial processing tree models 
It is well established that cognitive aging is associated with declines in episodic memory (Grady, 2012; Hedden \& Gabrieli, 2004). However, the size of age-related differences varies depending on the type and aspect of memory being considered. For example, normal aging is often accompanied by difficulties in remembering episodic details of prior events, whereas memory of the content of these events seems to be spared (e.g., Zacks \& Hasher, 2006). The Fuzzy-trace theory's (FTT) distinction between literal, precise memory representations (verbatim traces) and vague, meaning-based memory representations (gist traces), each of which evolves differently with aging, can account for this general pattern. According to the FTT, as we age, we are more likely to have trouble remembering specific, verbatim details than the general idea or "gist" of a given piece of information (e.g., Brainerd \& Reyna, 2004; Reyna \& Brainerd, 2011; Brainerd \& Reyna, 2015). Only a few studies have examined the effect of aging on the retrieval of verbatim and gist representations in recognition tasks (e.g., Castel, 2005; Gallo et al., 2019), with the vast majority of studies focusing on controlled and automatic retrieval processes such as recollection and familiarity (see Davidson \& Glisky, 2002; Yonelinas, 2002, for reviews). The present study aimed at examining the processes responsible for memory declines during normal aging by applying, for the first time in the literature, a state-of-the-art mathematical model that allows discriminating and precisely measuring the contribution of verbatim and gist memory in recognition performance.

Numerous studies investigated the contributions of recollection and familiarity to agerelated declines. In dual-retrieval theories, familiarity refers to circumstances in which subjects are confident that a test item is old, but they have no specific memory of its presentation. Recollection refers to being consciously aware of contextual details that accompanied test item's presentation (e.g., Parks et al., 2011; Rotello et al., 2004; Wixted \& Mickes, 2010). Two consistent findings have been obtained using paradigms such as Remember/Know, the process dissociation procedure and receiver operating characteristic 
analyses. On the one hand, there are substantial declines in recollection between the ages of 20 and 70, and on the other hand, declines in familiarity are smaller and statistically unreliable (Alghamdi \& Rugg, 2020; Koen \& Yonelinas, 2016). However, an ambiguous aspect of the definition of recollection is that it does not only imply that the subject remembers the target item itself, but also the details occurring at the same time as its presentation (Brainerd, Gomes et al., 2014; 2015). Hence, the question remains open whether age-related memory declines are specific to the recollection of contextual details or also affect the recollection of targetspecific information. Moreover, the impact of aging on the nature of the information retrieved via recollection or familiarity processes remains unclear (Guillaume et al., 2015; Tinard \& Guillaume, 2019). The major contributions of the FTT's verbatim-gist distinction are firstly, to characterize the nature of the traces retrieved, as a continuum between literal and meaningbased traces, and secondly, to distinguish between the retrieval of target-specific information and of contextual details, which are conflated in the conventional definition of recollection. Indeed, according to the FTT, context recollection can occur when either verbatim or gist traces are retrieved, because both are episodically tagged, but gist traces should not produce target recollection. Finally, familiarity occurs when gist traces are retrieved but stored contextual details do not come to mind (Brainerd, Gomes et al., 2014; 2015).

Building on this distinction, a model from the trichotomous theory of recall, which encompasses traditional dual-process distinctions has been proposed to account for memory changes that occur during aging (Brainerd, et al., 2009). This model was used to parse the processes responsible for late-life memory declines in healthy and impaired individuals. It was applied to recall tasks because the data used were from national studies in which these declines were measured with recall tasks (e.g., Langa et al., 2005). Brainerd and colleagues (2009; Brainerd \& Reyna, 2010) put forward that recall involves at least two distinct processes. Participants store verbatim and gist memory traces and, as the trials progress, they 
learn to recall them through a recollective retrieval operation that processes verbatim traces and a non-recollective one, referred to as reconstruction, that processes gist traces. Recollective retrieval allows direct access to verbatim traces of items (e.g., its identity or verbatim details such as its exact wording) without searching through traces of other items. It is a fast operation that produces errorless recall since surface forms of items are retrieved. Gist traces are used to retrieve the items via the reconstruction process. Gist traces are representations of partial identifying information of the items, especially semantic information such as "mountain" for the word "snow". The reconstruction operation uses gist traces to generate sets of items that match this information, for instance "snow" and "ski" for "mountain". These sets include true ("snow") and false ("ski”) candidates, making reconstruction more error-prone than recollection. Familiarity, a slave judgment operation of the reconstruction process, is assumed to perform confidence checks.

Brainerd and colleagues (2009; Brainerd \& Reyna, 2015) re-analyzed the data sets from national studies in which older adults (mean age $=70-80)$ performed standard recall tasks in which trials consist of alternating study cycles and memory tests, to estimate the contribution of both type of processes. These data were compared to data collected from younger adults. These processes were measured at the start of experiments and later on. In accordance with studies on recollection and familiarity, there was no decline in familiarity judgment with age, whether it was measured at the start of learning or later on. Direct access, i.e., the recollective retrieval operation, declined markedly with age, but this decline was concentrated in learning after the first trial, direct access being comparable between older and younger adults at the start of learning. Reconstruction also declined with age and this decline was also concentrated after the first trial.

The finding that, in recall tasks, familiarity judgment appears to be unaffected by aging is consistent with classic findings on familiarity in recognition tasks. As stated above, 
recollection in dual-retrieval theories includes both the recollection of target-specific information and of contextual information and it is unclear whether aging impacts one, the other or both. The results obtained on direct access shed some light on this issue because direct access involves retrieving item's verbatim trace, i.e., target recollection. They clearly indicate that direct access declines with age. Moreover, other studies showing an increase in false memories with age (e.g., Koutstaal \& Schacter, 1997; Lavoie \& Faulkner, 2000; Tun, et al., 1998) suggest that target recollection declines during aging while context recollection, which supports false memories, may not be (or less) affected. Hence older adults' reduced ability to suppress false memories suggests substantial declines in target recollection (Brainerd, et al., 2014). Reconstruction, which can be akin to context recollection because of the use of gist traces, is also affected by age but to a lesser extent than direct access. This is in line with the fact that, on the one hand, semantic knowledge is relatively preserved from the effects of aging (Hay \& Jacoby, 1999; Jennings \& Jacoby, 1993) but, on the other hand, reconstruction is an effortful process requiring executive resources to search in memory for candidate items that match gist traces and to select between them (Bugaiska et al., 2007).

Few studies have examined the effect of aging on the representations that are retrieved via recollective and non-recollective processes in recognition tasks. FTT assumes that gist and verbatim representations are retrieved independently through reconstruction and direct access, respectively. To empirically separate the two types of representations, Brainerd et al. (1999) proposed the conjoint recognition $(\mathrm{CR})$ paradigm and multinomial model. In a recognition test, when a match is found between a verbatim trace that stores perceptual details of each item, and the verbatim information of the probe, an identity judgment is made that leads to the acceptance of the probe. A detection of similarity between a gist trace that stores the core meaning of each item and the gist of the probe is also assumed to lead to an acceptance response. A gist-based similarity judgment can also underlie false recognition of semantically 
related distractors. This process is in opposition with a verbatim-based process, recollectionrejection (recall-to-reject), that underlies rejection of related distractors. This latter process is assumed to be based on a judgment of nonidentity between the verbatim information of the related distractor and the verbatim trace of the corresponding target. Hence, it reduces false recognition based on the retrieval of item-specific verbatim traces (Brainerd et al., 2003; Brainerd et al., 2014).

Previous findings suggested an age-related increase in reliance on gist-based retrieval processing that could explain why older adults make more false recognition of semantically related lures than younger adults (e.g., Devitt \& Schacter, 2016; Koustaal \& Schacter, 1997; Tun et al., 1998). Moreover, studies showed a decrease in older adults' accuracy in recalling verbatim details whereas the retrieval of gist-based information is less affected by age. Gallo, et al. (2019), for instance, showed younger and older adults information about the weather forecast for the week. Participants were then asked to recall forecast details of each day of the week (the verbatim details) or to recall which day(s) that week would be best to have a picnic and to bring an umbrella (the gist). The older adults were just as likely as the younger adults to remember what the best days were to go picnicking or to take an umbrella, but they were less likely to recall the verbatim details of each day forecast. Older adults also use gist memory to state whether a grocery item was overpriced, underpriced, or priced at market value (Castel, 2005) or to judge which of two comparable grocery items was the better buy (Flores et al., 2017). Taken together, these findings are consistent with the marked decline in direct-access and the lower decline in reconstruction observed in recall tasks. However, none of these studies empirically separated the respective contribution of verbatim and gist traces within a single task.

In the present study, we used the simplified version of the CR paradigm (Stahl \& Klauer, 2008) to estimate verbatim and gist memory traces within a single recognition task. 
Participants are presented with targets (i.e., items from the study list), related distractors (i.e., items that had not been presented on the study list but are related to a target via common gist) and unrelated distractors (i.e., new items that were neither part of the study list nor are related to a target). They are informed of the type of items on the test list and are asked to categorize each one as a target item, a related or an unrelated distractor. Performance in the CR memory test is determined by the interplay of verbatim and gist memory processes as well as by guessing. As explained above, in case of available verbatim memory, a target probe is identified as target via an identity judgment between the target's verbatim trace and the target probe and a related distractor is identified as a related item via a nonidentity judgment between the target's verbatim trace and the related distractor. Given no verbatim memory but available gist memory, participants have identified the target or related probe's meaning as old but cannot remember whether the probe itself or a related item with the same gist had been presented in the learning phase. At this time, they have to decide between target and related responses which is modeled by a guessing process. Classification of unrelated probes are also based on a combination of guessing processes in this model.

We manipulated the activation strength of gist memory to examine the effect of aging on recognition performance as a function of the ease of retrieving gist traces of studied items. Gist memory for a concept or theme was manipulated by presenting one versus multiple items related to that theme at study. When a theme is repeatedly activated at study by multiple items, memory for its gist is increased. This manipulation has been proven effective in affecting gist memory parameters in the original and the simplified CR paradigm (Brainerd et al., 1999; Brainerd, et al., 2001; Stahl \& Klauer, 2008). We used lists of semantic associates converging on a common theme (e.g., "honey, hive, wasp, hornet"- "bee"), such as Deese- 
Roediger-McDermott lists (DRM, Deese, 1959, Roediger \& McDermott, 1995) ${ }^{1}$. The characteristic of these lists is that there are strong associations among list items and the theme word of each list. By presenting an increasing number of list items, gist memory of the theme of the list as well as of other list items becomes increasingly activated.

Previous studies (e.g., Brainerd et al., 2009) showed that, in recall tasks, direct access and also, but to a lesser extent, reconstruction are affected by age while the judgment of familiarity is completely spared. Other studies (e.g., Gallo et al., 2019) suggested that older adults have difficulty performing tasks that require them to retrieve the studied items in detail, but that they are just as successful as younger adults in retrieving the gist of these items. Although gist and verbatim memory were estimated indirectly in these studies, their findings led us to predict a decrease in verbatim memory whereas gist memory should be preserved with increasing age in the CR paradigm. Recently, a study using this paradigm to estimate verbatim and gist memory in older adults focused on the memory of associations between pairs of images (Greene \& Naveh-Benjamin, 2020). Results showed a decrease in verbatim memory with age, whereas gist memory was unaffected.

Finally, if, as suggested by previous studies, gist memory is preserved from the effects of aging while verbatim memory is affected, the effect of age on correct recognition should be greater in the weak than in the strong gist activation condition. Indeed, both younger and older adults should rely more on gist memory to identify target and related probes in the strong than in the weak gist activation condition. However, older adults should have greater difficulty than younger ones in identifying target and related probes in the weak gist activation condition in which they should be less able to retrieve gist traces of the items. Conversely, if

\footnotetext{
${ }^{1}$ Thematic associations were chosen over taxonomic associations because studies have shown that recall of the former is less impacted by age than the latter (e.g., Belacchi \& Artuso, 2018). This reduces stimulus-related intergroup bias.
} 
both gist and verbatim traces decline with age, then younger adults should perform better than older adults regardless of gist activation.

\section{Method}

\section{Participants}

Sixty-three young adults $(M=20.4, S D=1.35$, range $=18-24$ years, 54 females $)$ and 46 older adults $(M=67.7, S D=4.95$, range $=61-76$ years, 28 females $)$ were recruited from Aix-Marseille University and the surrounding community. Participants were native French speakers. All participants reported to be in good health, had normal or corrected-to-normal hearing and vision. The Mini Mental State Examination (MMSE; Folstein, Folstein, \& McHugh, 1975) was administered to them to screen for signs of abnormal cognitive decline. All participants scored above the standard cut-off of 26 points on the MMSE $(M=28.8, S D=$ 1.28). This study was carried out in accordance with the recommendations of the APA Ethics Code. All subjects gave written informed consent in accordance with the Declaration of Helsinki. The institutional review board of Aix-Marseille University declares that the study does not raise any ethical or regulatory issues. As Bayesian analyses were used, we did not conduct a power-analysis given that evidence for a null effect is equally informative in the Bayesian framework (Kruschke, 2011; 2018) and the chance of making a Type 1 error did not increase with optional stopping (Rouder, 2014). We decided to use a sample size of at least twice the sample sizes than in the original simplified CR paradigm $(N=20$; Stahl \& Klauer, 2008) per age group due to the addition of the older adult group in which there could be greater variability. After data collection, we used a Bayesian prior sensitivity analysis to test whether the results are robust across different prior specifications (Greene \& NavehBenjamin, 2020). We specified the multinomial processing tree model with priors varying across three levels of informativeness. The parameter estimates of verbatim and gist memory 
and guessing processes were stable (i.e., robust) across prior specifications (see the Bayesian prior sensitivity analyses in the OSF).

\section{Materials}

Lists of words were taken from the verbal association norms for concrete French nouns (Bonin et al., 2018). Such as DRM lists, (each list consists of several words (e.g., "honey, hive, wasp, hornet, etc.") that are all associated with a common theme or gist (e.g., "bee"). Gist memory was defined as memory for the list theme, and verbatim memory was defined as memory for the presented list words. Sixty lists consisting of four words and one theme word were selected (see the word lists in the OSF). The mean backward association strength between each theme word and its associates were fairly high $(M=71.8, S D=0.15)$. Lists were randomly assigned to contribute either as a target, a related distractor, or an unrelated distractor to the test list. The first words of presented lists were used as targets, the theme words were used as related distractors, and the first words of unpresented lists were used as unrelated distractors.

Gist activation was manipulated by presenting different numbers of words from each theme on the study list. In the weak gist condition, a theme (e.g., "circus") was represented by a single related word (e.g., "clown"). In the strong gist condition, a theme (e.g., "bee") was represented by four related words (e.g., "honey, hive, wasp, hornet”). The study list comprised words from 40 randomly selected lists that were randomly split into two halves of 20 lists each. Lists from the first halve were represented by the first list word. Lists from the second half were represented by the first four words. In total, 100 words were thereby presented in random order in the study phase.

At test, the 20 single-word lists, and the 20 four-word lists were randomly split into 10 lists for which the first list word was shown as target probe and 10 lists for which the theme word was shown as related distractor. Unrelated distractors were the first list words from the 20 
lists that were not presented in the study list. In total, 60 words were thereby presented at test in random order. All randomizations were carried out for each participant anew. To avoid tiring the subjects, the memory task was separated into two sessions. In each session, 50 words were presented in the study phase and 30 words were presented at test.

\section{Procedure}

There were two testing sessions in the study. In each session, the simplified CR procedure was used (see Figure 1). The task was similar across the two sessions except the words used. Among the 100 selected words, 50 were studied and tested in the first session and 50 in the second session. The two sessions were separated by a break that could go up to half a day to avoid too much fatigue and interference between both sessions. The break was shorter (e.g., 20-30 min) for the younger group to prevent experimental mortality.

At the beginning of each session, participants were instructed that a list of words would be presented and that they had to remember this list for a later test. Memory words from the strong and the weak gist activation condition were presented randomly and sequentially for $3000 \mathrm{~ms}$ followed by a $300 \mathrm{~ms}$ interstimulus interval (ISI). They were displayed in black letters on a medium gray background. After the study phase, participants spotted the differences between two pictures for a total duration of $5 \mathrm{~min}$. Next, they were sequentially presented with the list of probes of the recognition task consisting of $1 / 3$ of targets, $1 / 3$ of related distractors and $1 / 3$ of unrelated distractors. For each probe, they had to indicate whether it was identical to a word of the study list (i.e., a "target"), "related" to a word of the study list or "new" by pressing the appropriate key.

A training phase preceded each experimental session. Participants completed the same memory task than in the experiment but with only six words. They were familiarized with the different probe types (i.e., target, related and unrelated distractors) of the memory test. 
Finally, they could decide to start the training again as many times as they wished. At the end of the experiment, participants were thanked, debriefed, and dismissed.

\section{Figure 1}

Diagram of the experimental procedure

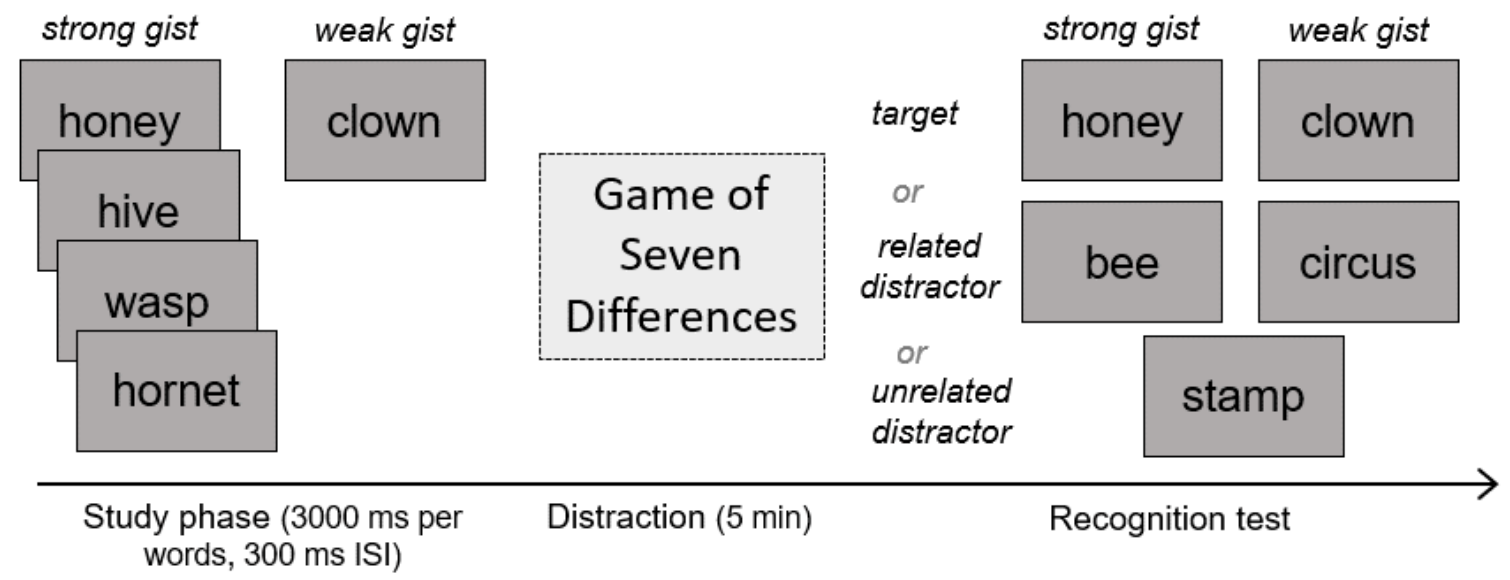

Results

The data and additional Null Hypothesis Significance Testing (NHST) analyses are available at the OSF.

\section{Memory accuracy}

Analysis plan. Default Bayesian mixed-measures ANOVA (Rouder, et al., 2012) were conducted separately for each probe type (target, related and unrelated probes) with gist activation (weak vs. strong) and response type (target vs. related vs. unrelated) as withinsubject factors and age group (younger vs. older adults) as a between-subject factor. The analyses were conducted using JASP version 0.11.1.0 (JASP Team, 2019). In Bayesian hypothesis testing, the strength of evidence for a specified model (M1) was quantified by comparing this model against a null or reduced model (M0). The ratio of the likelihood of the two models under comparison is the Bayes Factor $\left(\mathrm{BF}_{10}\right)$. The $\mathrm{BF}_{10}$ of each model was obtained by comparing it to the null model. First, we reported the best model, the model with 
the largest $\mathrm{BF}_{10}$. Then, we report the $\mathrm{BF}_{\text {inclusion }}$ value for each factor in the best model (i.e., a main effect or an interaction effect), which indicates the likelihood of the data under models that included a given factor compared to all models stripped of the factor. Strength of evidence is evaluated using Kass \& Raftery's (1995) interpretation of Bayes Factors. Target probes. A first Bayesian analysis was conducted on responses to target probes. Figure 2 shows the percentage of each response type to target probes as a function of gist activation and age group. The best model included main effects of response type and age group and the interaction between both variables $\left(\mathrm{BF}_{10}=2.29 \times 10^{199}\right)$. Table 1 shows the $\mathrm{BF}_{\text {inclusion values }}$ for each main effect and interaction. Regarding the main effect of response type, as expected, a post-hoc comparison indicated that target probes were decisively more often identified as targets $(71.8 \%, S D=16.0)$ than as related $(14.4 \%, S D=12.0)$ or unrelated $(13.8 \%, S D=$ 10.1) distractors $\left(\mathrm{BF}_{10}=2.69 \times 10^{70} ; \mathrm{BF}_{10}=3.84 \times 10^{75}\right.$, respectively $)$. There was strong evidence that the percentage of related and unrelated responses did not differ $\left(\mathrm{BF}_{10}=0.09\right)$.

\section{Figure 2}

Percentages of target, related, and unrelated responses to target probes as a function of gist activation and age group

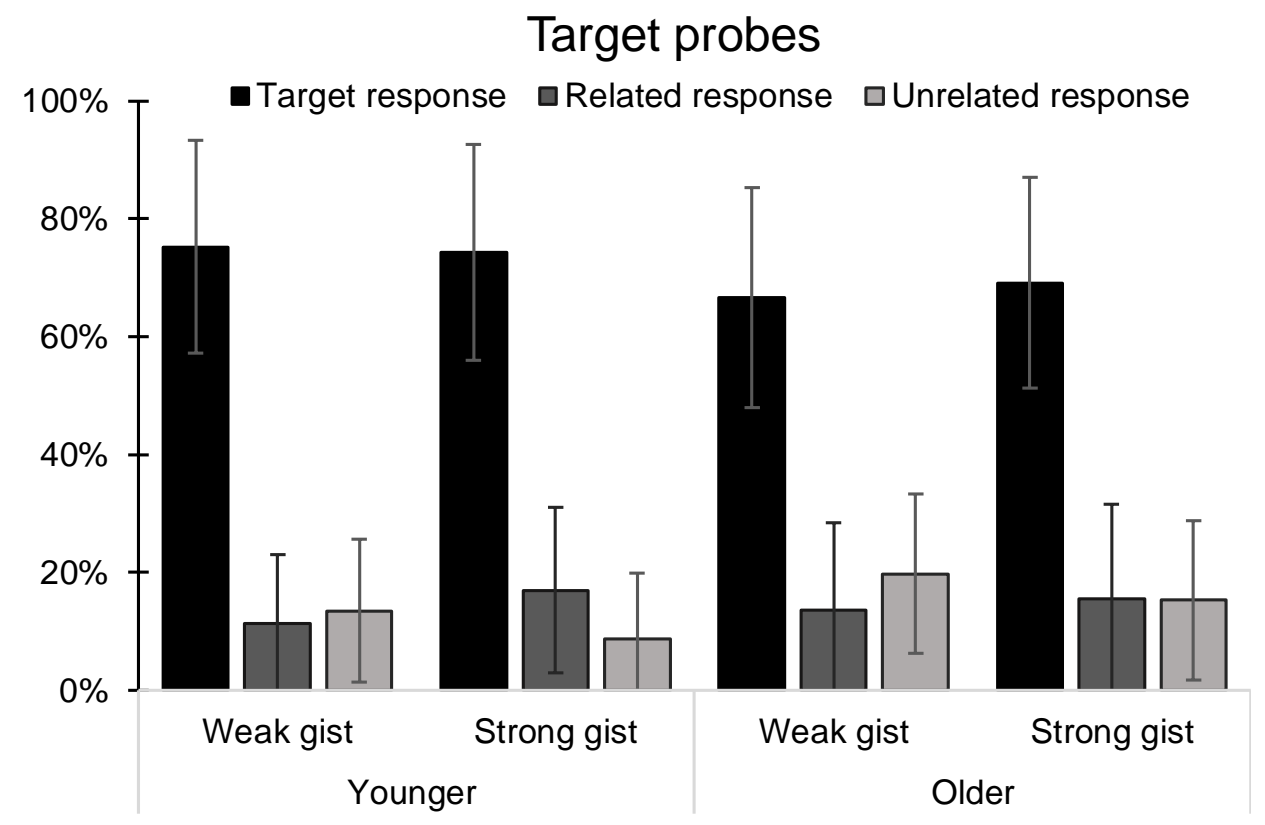


Note. Error bars represent standard errors.

To decompose the interaction between response type and age group, we conducted Bayesian independent samples t-tests separately for each response type with age group as grouping variable. As expected, although the evidence was weak, younger adults more often correctly judged target probes as such $(74.8 \%, S D=15.6)$ than older adults $(67.8 \%, S D=$ 15.9; $\mathrm{BF}_{10}=2.00$ ). The analysis provided substantial evidence against a difference between both age groups on the percentage of related responses $(14.1 \%, S D=11.1 ; 14.8 \%, S D=0.13$ for younger and older adults, respectively; $\mathrm{BF}_{10}=0.21$ ). Finally, as predicted, there was strong evidence that older adults $(17.4 \%, S D=10.4)$ made more misses than younger adults, i.e., they classified y target probes as unrelated distractors more often $(11.1 \%, S D=9.10$; $\left.\mathrm{BF}_{10}=25.9\right)$.

Related distractors. A second Bayesian analysis was conducted on response to related distractors (Figure 3). The best model included the main effects of the three variables and the interactions between gist activation and response type and between response type and age group $\left(\mathrm{BF}_{10}=9.10 \times 10^{92}\right)$. Regarding the main effect of response type, as expected, a posthoc comparison indicated that participants decisively identified related distractors as such $(52 \%, S D=21.1)$ more often than as targets $\left(9.7 \%, S D=11.8 ; \mathrm{BF}_{10}=4.11 \times 10^{44}\right)$ or unrelated distractors $\left(38.3 \%, S D=17.7 ; \mathrm{BF}_{10}=1023\right)$. Related distractors were also identified more often identified as unrelated distractors than as targets $\left(\mathrm{BF}_{10}=4.52 \times 10^{33}\right)$.

\section{Figure 3}

Percentages of target, related, and unrelated distractor responses to related distractors as a function of gist activation and age group 


\section{Related distractors}

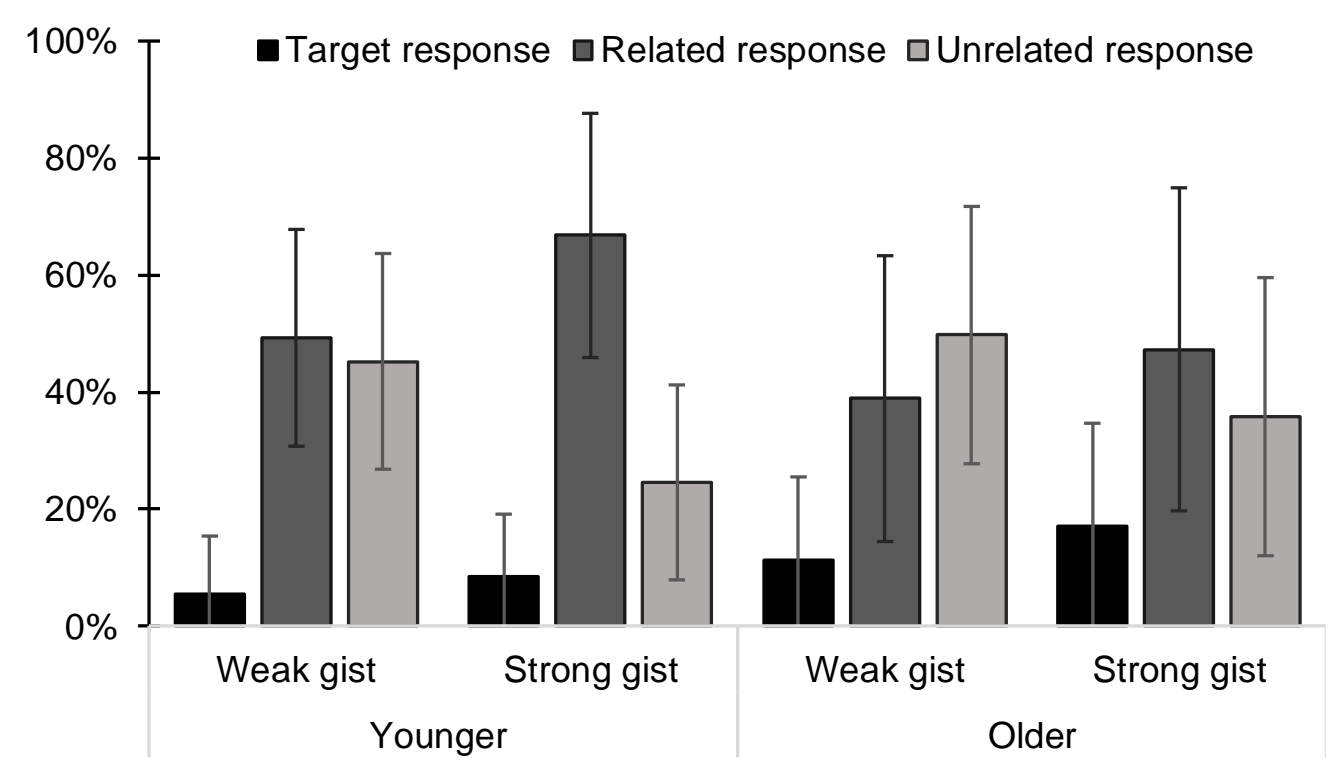

Note. Error bars represent standard errors.

To decompose the interaction between gist activation and response type, Bayesian paired ttests were conducted separately for each response type with gist activation as a repeatedmeasure. As predicted, related distractors were decisively more often correctly identified as related distractors $\left(\mathrm{BF}_{10}=6.28 \times 10^{6}\right)$ and strongly more often falsely identified as targets $\left(\mathrm{BF}_{10}=54.4\right)$ in the strong $(58.8 \%, S D=25.6$ for the response "related" and $11.8 \%, S D=$ 14.3 for the response "target") relative to the weak gist activation condition $(45.1 \%, S D=$ 21.6 for the response "related" and $7.6 \%, S D=12.1$ for the response "target"). By contrast, they were more often identified as unrelated distractors $\left(\mathrm{BF}_{10}=2.50 \times 10^{12}\right)$ in the weak $(47.2 \%, S D=20.1)$ relative to the strong $(29.4 \%, S D=20.7)$ gist activation condition.

Finally, to decompose the interaction between response type and age group, Bayesian independent t-tests were conducted separately for each response type with age as grouping variable. As expected, younger adults were better at identifying related distractors as such $(58.0 \%, S D=16.5)$ than older adults $\left(43.7 \%, S D=23.9 ; \mathrm{BF}_{10}=73.8\right)$. Older adults more often falsely identified them as targets $\left(13.4 \%, S D=14.1 ; \mathrm{BF}_{10}=6.90\right)$ or unrelated 
distractors $\left(42.9 \%, S D=21.3 ; \mathrm{BF}_{10}=2.51\right)$ than younger adults $(7.1 \%, S D=9.1 ; 34.9 \%, S D$ $=13.8$, for "target" and "unrelated" responses, respectively).

Unrelated distractors. A third Bayesian analysis was conducted on response to unrelated distractors with response type as a within-subject factor and age group as a between-subject factor (Figure 4). The best model included main effects of both variables and the interaction between them, $\left(\mathrm{BF}_{10}=9.74 \times 10^{87}\right)$. Concerning the main effect of response type, as predicted, a post-hoc comparison indicated that unrelated distractors were decisively more often identified as such $(62.6 \%, S D=15.6)$ than as targets $\left(10.1 \%, S D=10.3 ; \mathrm{BF}_{10}=3.39 \times 10^{43}\right)$ or related distractors $\left(27.3 \%, S D=15.1 ; \mathrm{BF}_{10}=1.41 \times 10^{20}\right)$. They were also decisively more often classified as related distractors than as targets $\left(\mathrm{BF}_{10}=1.97 \times 10^{11}\right)$.

\section{Figure 4}

Percentages of target, related, and unrelated distractor responses to unrelated distractors as a function of age group

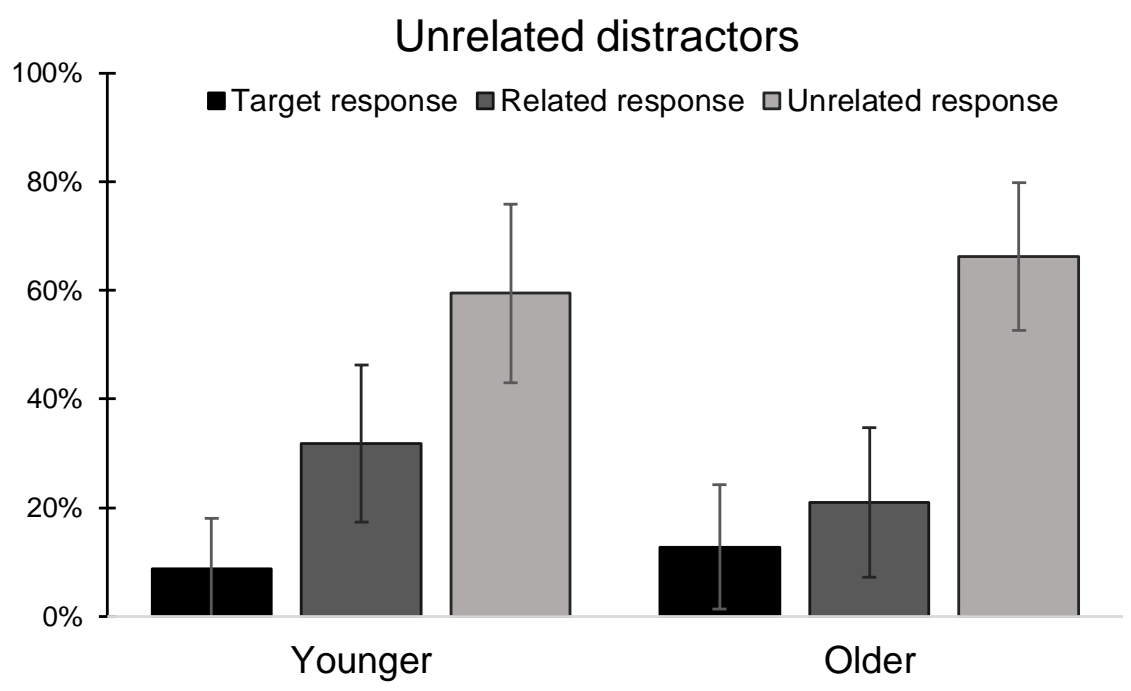

Note. Error bars represent standard errors.

To decompose the interaction between response type and age group, Bayesian independent samples t-tests were conducted separately for each response type with age as a grouping variable. Unexpectedly, older adults $(67 \%, S D=13.6)$ were better than younger adults 
$(59.4 \%, S D=16.4)$ at identifying unrelated distractors as such $\left(\mathrm{BF}_{10}=3.48\right)$. By contrast, younger adults $(31.8 \%, S D=14.5)$ more often identified them as related distractors than older adults $\left(21.1 \%, S D=13.7 ; \mathrm{BF}_{10}=141\right)$. Finally, the percentage of target responses was not affected by age group $(8.7 \%, S D=9.3 ; 12 \%, S D=11.4$ for younger and older adults, respectively, $\left.\mathrm{BF}_{10}=0.66\right)$.

\section{Table 1}

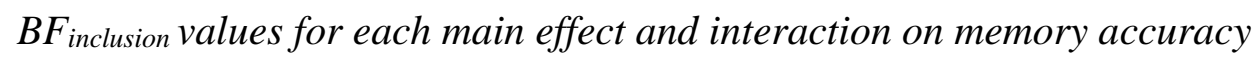

\begin{tabular}{|c|c|c|c|}
\hline Probe type & Effects & $\mathrm{BF}_{\text {inclusion }}$ & Evidence \\
\hline \multirow{7}{*}{ Target } & Gist activation & 0.09 & strong against \\
\hline & Response type & $5.96 \times 10^{197}$ & decisive \\
\hline & Age group & 0.002 & decisive against \\
\hline & Gist activation $\times$ Response type & 2.32 & weak \\
\hline & Gist activation $\times$ Age group & 0.17 & substantial against \\
\hline & Response type $\times$ Age group & 407 & decisive \\
\hline & $\begin{array}{l}\text { Gist activation } \times \text { Response type } \times \text { Age } \\
\text { group }\end{array}$ & 0.07 & strong against \\
\hline \multirow{7}{*}{ Related } & Gist activation & 0.08 & strong against \\
\hline & Response type & $2.74 \times 10^{74}$ & decisive \\
\hline & Age group & 0.08 & strong against \\
\hline & Gist activation $\times$ Response type & $1.00 \times 10^{14}$ & decisive \\
\hline & Gist activation $\times$ Age group & 0.13 & substantial against \\
\hline & Response type $\times$ Age group & $3.84 \times 10^{7}$ & decisive \\
\hline & $\begin{array}{l}\text { Gist activation } \times \text { Response type } \times \text { Age } \\
\text { group }\end{array}$ & 0.53 & weak against \\
\hline Unrelated & Response type & $1.07 \times 10^{85}$ & decisive \\
\hline
\end{tabular}




$\begin{array}{lcc}\text { Age group } & 0.13 & \text { substantial against } \\ \text { Response type } \times \text { Age group } & 7762 & \text { decisive }\end{array}$

Note. Strength of evidence is evaluated using Kass and Raftery's (1995) interpretation of Bayes Factors.

\section{Verbatim and gist memory}

The simplified conjoint recognition model. The multinomial processing tree (MPT) model of the simplified CR paradigm (Stahl \& Klauer, 2008) depicted in Figure 4 was used to compute parameter estimates for verbatim $\left(V_{t}\right)$ and gist memory for targets $\left(G_{t}\right)$, for verbatim $\left(V_{r}\right)$ and gist memory for related distractors $\left(G_{r}\right)$ and for guessing processes $(a$ and $b)$. MPT models attempt to explain through which processes (circles) participants give a response (boxes on the right) to a memory probe (boxes on the left). The probability of giving a response $i$ to a probe $j$ is estimated by summing the probabilities of each branch leading to this response.

The two parameters $V$ represent the probability of retrieving a verbatim trace of a target when a target probe $\left(V_{t}\right)$ or a related distractor $\left(V_{r}\right)$ is presented at test. The two parameters $G$ correspond to the probability that participants retrieve a gist trace of a target for a target probe $\left(G_{t}\right)$ or a related distractor $\left(G_{r}\right)$, given that they have not retrieved its verbatim trace. When participants retrieve gist memory, they cannot remember whether the probe itself or a related word with the same gist was presented at study. They then guess whether the probe is a target (with probability $a$ ) or a related distractor (with probability $1-a$ ). When neither verbatim nor gist is available, a participant can still guess that the probe meaning is old with the probability $b$. The decision between the "target" and "related" responses is again modelled by the parameter $a$. Otherwise, the participant guesses that the probe is new with the probability $1-$ $b$. Verbatim and gist traces do not intervene in the responses to unrelated distractors because 
these probes do not trigger the retrieval of verbatim or gist representations of the study phase.

Therefore, the responses to unrelated distractors are based entirely on $b$.

\section{Figure 5}

Multinomial processing tree for the simplified conjoint recognition paradigm based on Stahl and Klauer (2008)

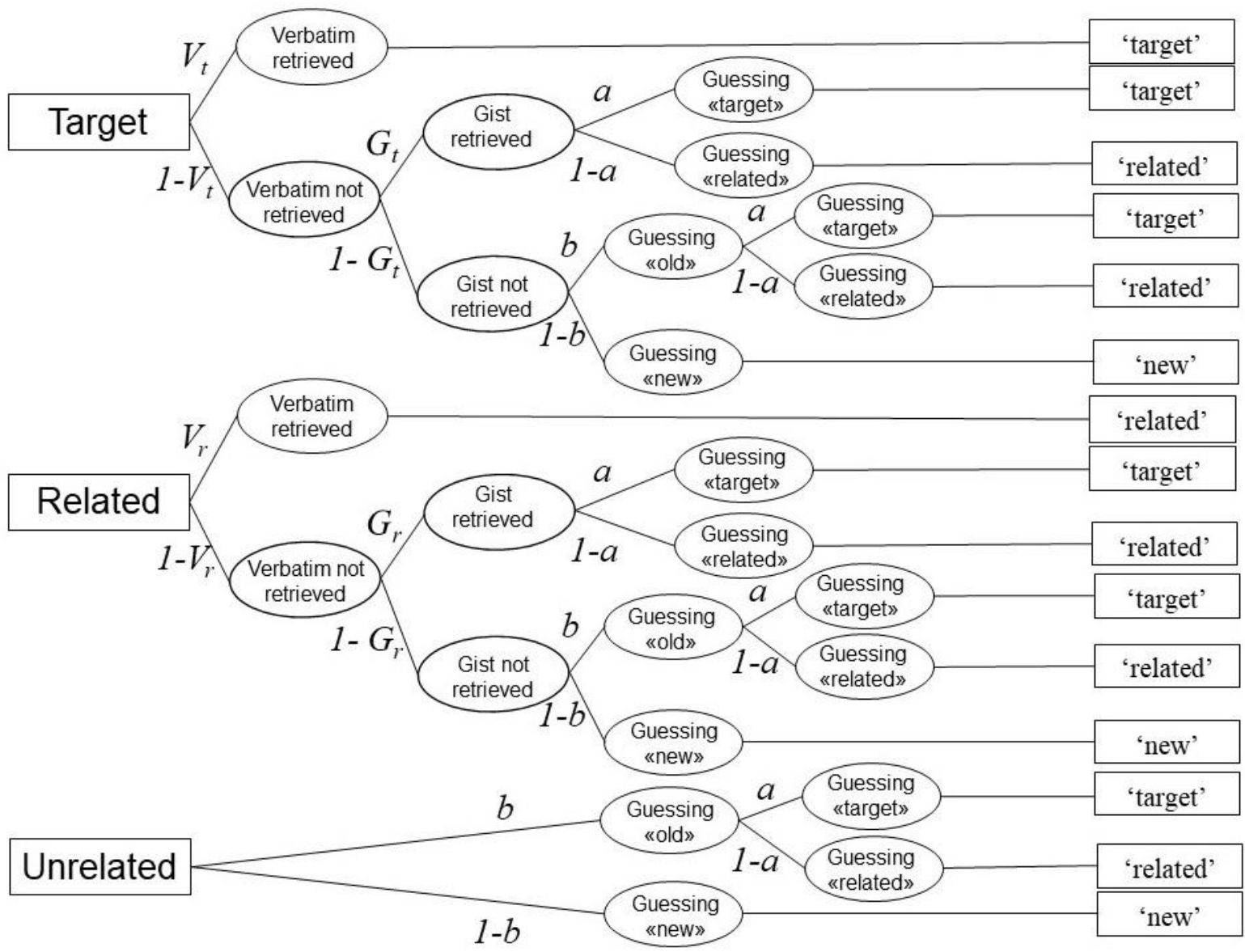

Note. $V_{t}=$ probability of retrieving a target's verbatim trace given a target probe; $V_{r}=$ probability of retrieving a target's verbatim trace given a related probe; $G_{t}=$ probability of retrieving a target's gist trace given a target probe; $G_{r}=$ probability of retrieving a target's gist trace given a related probe; $b=$ probability of guessing that an item is either a target or a related probe; $a=$ probability of guessing "target".

Verbatim, gist and guessing parameter estimates. Verbatim, gist and guessing parameter estimates are given in Table 2. Guessing parameters, $a$ and $b$ were set equal across the gist 
activation factor for identifiability reasons ${ }^{2}$. We fitted the model separately to both age groups (the results of the model fitting are available on the OSF), with hierarchical latent-traits priors specified using the TreeBUGS package for R (Heck, Arnold, \& Arnold, 2018; R Core Team, 2019). We adapted the analysis script for MPT developed by Greene and Naveh-Benjamin (2020, available at https://osf.io/xdhgz/).

\section{Table 2}

Parameter estimates for verbatim and gist memory and guessing processes as a function of gist activation and age group

\begin{tabular}{ccccc}
\hline \multirow{2}{*}{ Parameters } & \multicolumn{2}{c}{ Younger } & \multicolumn{2}{c}{ Older } \\
\cline { 2 - 5 } & Weak gist & Strong gist & Weak gist & Strong gist \\
\hline$V_{t}$ & $.74[.68, .80]$ & $.71[.65, .77]$ & $.59[.51, .67]$ & $.61[.51, .69]$ \\
$V_{r}$ & $.22[.13, .30]$ & $.30[.11, .46]$ & $.15[.04, .26]$ & $.08[.004, .20]$ \\
$G_{t}$ & $.18[.03, .33]$ & $.55[.39, .69]$ & $.25[.06, .41]$ & $.44[.25, .59]$ \\
$G_{r}$ & $.03[.001, .11]$ & $.35[.18, .51]$ & $.07[.003, .19]$ & $.40[.24, .53]$ \\
$b$ & $.39[.35, .44]$ & $.33[.28, .37]$ \\
$a$ & \multicolumn{1}{c}{$.17[.12, .23]$} & $.32[.21, .44]$ \\
\hline
\end{tabular}

Note. Parameter estimates are group level means, and 95\% Bayesian credible intervals are in brackets.

For each parameter, we computed a credibility interval of the difference between gist activation conditions and between age groups. The posterior samples obtained in the weak gist condition were subtracted from those obtained in the strong gist condition and those of the older adults were subtracted from those of the younger adults. The differences are shown in Figure 5. Parameters for which the $95 \%$ CI of the difference estimates overlapped with 0 do

\footnotetext{
${ }^{2}$ The manipulation of gist activation affected only targets and related distractors but not unrelated distractors. Therefore, the model has more parameters (12) than degree of freedom in the data (10 independent empirical probabilities), making it necessary to equate two parameters across gist activation condition. We imposed the equality restriction on the guessing parameters as gist activation did not impact responses to unrelated probes (see the model equations in the appendix of Stahl and Klauer, 2008).
} 
not meaningfully differ (Smith \& Batchelder, 2010). As expected, there were higher values of gist memory parameters $\left(G_{t}\right.$ and $\left.G_{r}\right)$ in the strong than in the weak gist condition in the younger adults group. Gist memory for related distractors was specifically increased in the strong gist condition in the older adults group. Verbatim memory parameters $\left(V_{t}\right.$ and $\left.V_{r}\right)$ were not affected by gist activation. As predicted, in both gist activation conditions, younger adults exhibited higher values of verbatim memory for targets but did not differ from older adults on gist parameters $\left(G_{t}\right.$ and $\left.G_{r}\right)$. Younger adults had a greater tendency to guess that an item's meaning is old $(b)$ than older adults. The latter's greater reverse tendency to guess that an item's meaning is new $(1-b)$ could explain their greater ability to identify unrelated distractors as such. The latter, when they identified an item meaning as old either on the basis of guessing or of gist memory, were more likely to identify it as a target rather than a related distractor $(a)$. It was especially the case in the weak gist condition.

\section{Figure 5}

Forest plots depicting the difference scores. 
a

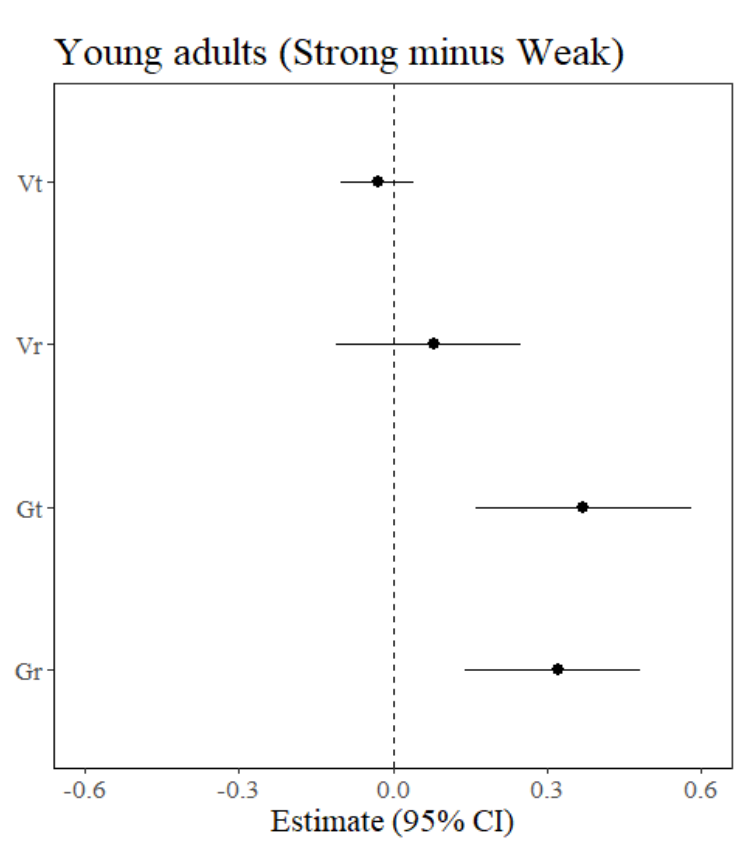

c

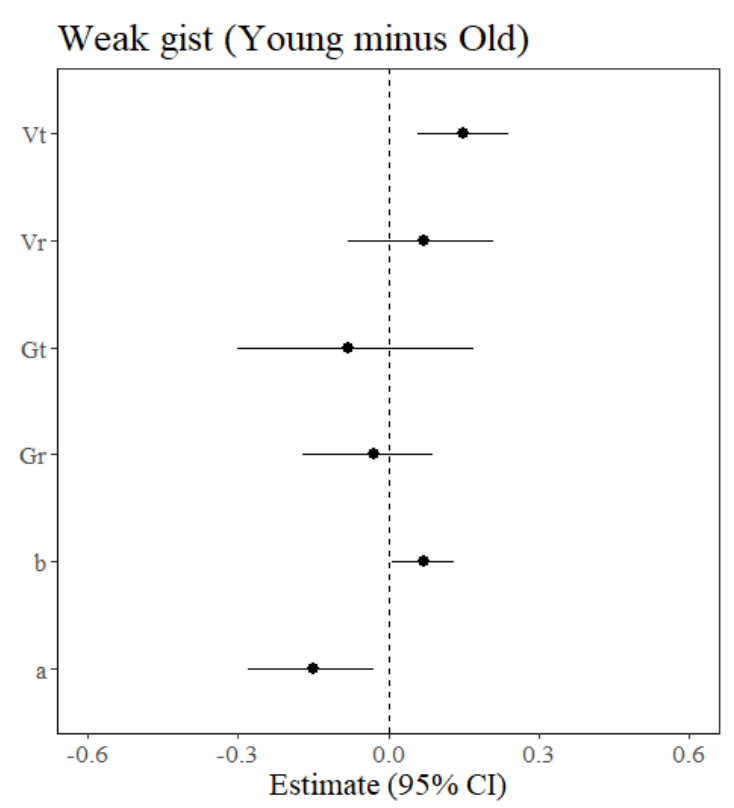

b

Older adults (Strong minus Weak)

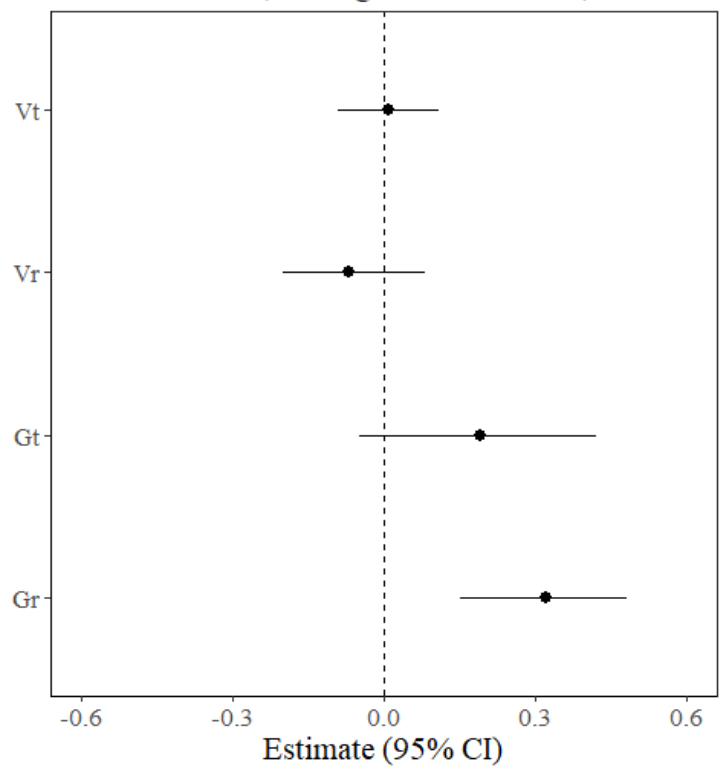

d

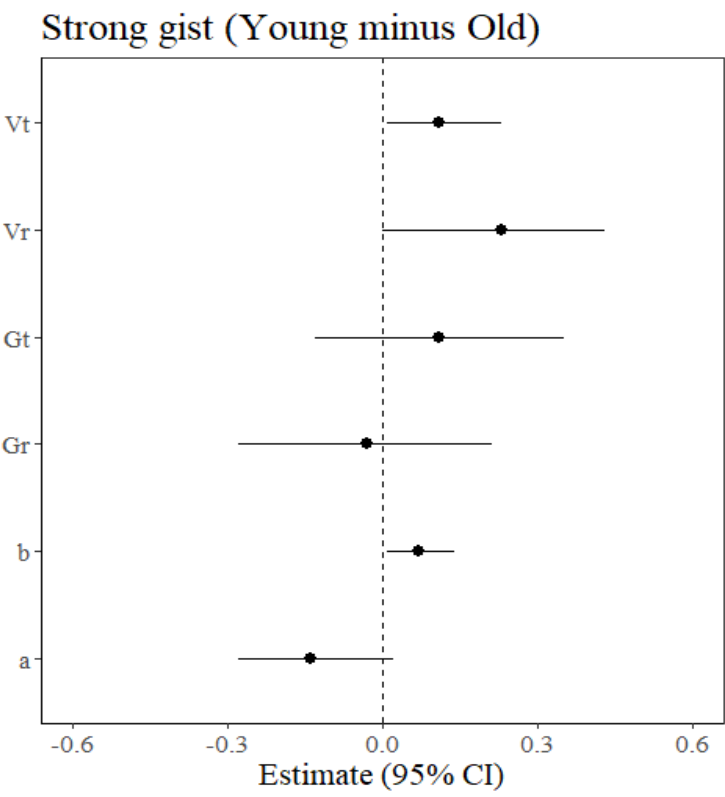

Note. Forest plots depicting, for each parameter, the difference scores between the strong and weak gist conditions for younger (a) and older adults (b) and between younger and older adults in the weak (c) and strong gist (d) conditions. Points indicate the posterior mean difference, lines the $95 \% \mathrm{CI}$ for the difference score. Dashed line at 0 indicate a point null.

\section{General Discussion}


Guided by the principles of the FTT (Brainerd \& Reyna, 2015), we have shown that older adults have deficits, relative to younger adults, in retrieving the verbatim traces of items per se but they can retrieve their semantic features or gist. Previous studies have indicated that aging affects recollective retrieval more than non-recollective retrieval, the former allowing the retrieval of verbatim traces and the latter of gist traces (e.g., Brainerd et al., 2009). Other studies suggested that older adults preferentially rely on gist-based processing strategies (e.g., Koustaal \& Schacter, 1997; Gallo et al., 2019). Our study goes beyond the previous ones because we adopted a multinomial modeling approach that provided a fine-grained measure of the respective contribution of verbatim and gist representations to responses to a single recognition task.

First, results indicated that the effects of aging on the ability to retrieve verbatim and gist traces are clearly dissociated. Verbatim parameters for targets were substantially affected by age. Although verbatim parameters for related distractors were lower and more variable than those for targets, we nevertheless observed that older adults retrieved verbatim traces enabling them to reject related distractors $(11,5 \%$ of cases) less than half as often as younger adults ( $26 \%$ of cases). Thus, verbatim retrieval appears to decrease with age while there is no general age-related trend in gist retrieval. In addition, despite a clear decline with age in the ability to retrieve verbatim traces, verbatim memory for targets remained fairly good in older adults. Moreover, the ability to retrieve verbatim memory when a target was presented at test was much better at both age levels than the ability to retrieve verbatim memory when a related distractor was presented. This is a classic response pattern predicted by the FTT (Brainerd et al., 1999); targets are better retrieval cues for their own surface forms than related distractors. Second, results showed that the ability to retrieve verbatim memory and the ability to retrieve gist memory were also dissociated. Older adults, as well as younger ones, made more use of gist representations in the strong gist than in the weak gist activation condition, 
but gist activation had no effect at all on verbatim retrieval. This is particularly the case for related distractors that both younger and older adults recognized more often either as related or target items based on gist traces in the strong (25\% and $37 \%$ of cases, respectively) than in the weak ( $2 \%$ and $6 \%$ of cases, respectively) gist activation condition. Younger adults also mobilized more gist representations to respond to targets in the strong gist condition (16\% of cases versus only $5 \%$ of cases in the weak gist condition). The difference among the two conditions, although still present, was less marked for older adults who used gist traces $17 \%$ of the cases in the strong gist condition versus $10 \%$ of the cases in the weak gist condition.

The question remains as to why the recognition performances of older adults were still lower than those of younger adults in the strong gist condition in which, as expected, gist representations contribute the most to the recognition judgments. When looking at the probability of classifying target probes as targets or related distractors based on gist memory, it turns out that both younger and older adults tend to identify them more often as related distractors (13\% and $12 \%$ of the cases, respectively) rather than as targets (3\% and $5 \%$ of the cases, respectively). Verbatim memory for targets was nevertheless greater among younger than older adults which explains why target recognition performance of the former was still better in the strong gist activation condition. A manipulation more strongly targeting gist memory for targets, such as presenting words belonging to the same category rather than DRM-like lists (Brainerd et al., 2001; Stahl \& Klauer, 2008), may be more beneficial for older adults who may use gist memory more often to correctly recognize targets. Concerning related probes, gist retrieval led younger adults in the strong gist activation condition to classify related probes correctly in $20 \%$ of cases and as targets in only $4 \%$ of cases. Gist retrieval also resulted in more correct identifications of related items $(25 \%)$ in older adults but also in false recognition of these items as targets, in a significant number of cases (12\%). Younger adults could also rely more often than older adults on verbatim representations to 
correctly classify related items, again reflecting the better recognition performance of the former. However, it is interesting to note that when older adults correctly identified a related item in the strong gist activation condition, it was mostly via gist memory (25\% of cases, $8 \%$ of cases via verbatim memory and $12 \%$ of cases via guessing) whereas it was mostly via verbatim memory in younger adults (30\% of cases, $20 \%$ of cases via gist memory and $15 \%$ of cases via guessing). This finding suggests that older adults may be able to use gist memory to retrieve presented (verbatim) information despite losses in verbatim memory. This is a functional coping strategy that would be of great interest to explore further in future research. Although our manipulation of gist memory activation proved effective, an experiment more strongly manipulating gist activation, such as blocking the words that follow the same theme together in consecutive study positions (e.g., Brainerd et al., 2008), might produce sharper differences to related distractors among older adults.

Our findings show that representations contain less detail, they become fuzzier and are more based on general meaning during aging. Reliance on gist memory enabled older adults to correctly remember a number of items but also to generate more memory errors, such as semantic false memories in the strong gist condition. This explains why even in this condition, their recognition performance did not equal that of younger adults. Therefore, our findings are congruent with FTT's predictions.

Our results also add to evidence concerning the hypothesis that aging is associated with deficits in associative processes that are responsible for binding the information content of items to their sources or contexts (Naveh-Benjamin, 2000; Naveh-Benjamin, Hussain, Guez \& Bar-On, 2003). According to the associative deficit hypothesis, older adults can remember individual components of an episode relatively well, compared to younger adults, but often fail to encode or retrieve the associations among these components. This failure contributes to memory errors such as misremembering where a person was previously 
encountered (Chen \& Naveh-Benjamin, 2012). Drawing on this principle, Greene \& NavehBenjamin (2020) recently demonstrated that older adults can remember the gist of associations, that is associations at lower levels of specificity, to relatively similar extents as younger adults but they fail to retrieve associative episodic memories at highly specific levels of representation. Taken together, Greene and Naveh-Benjamin's results and the decrease in verbatim memory for targets observed in older adults in the present study confirm that specific information retrieval is particularly impaired in aging. In addition, the difference between younger and older adults in correct recognition of targets was twice as larger in the weak gist condition requiring more specific information retrieval relative to the strong gist condition. By contrast, in terms of correct identification of related distractors, the difference between the two age groups is twice as great in the strong gist condition, which is due to the fact that older adults made more false memories in this condition. This is an additional argument in favor of an age-related decrease in recollection of target-specific information. Finally, our results also support studies showing that memory deficits during aging primarily concern the ability to reduce interference (e.g., Pettigrew, \& Martin, 2014; Reyna, \& Mills, 2007) and to process pattern separation (e.g., Burke et al., 2010) rather than recollection and controlled retrieval processes in general (e.g., West, 1996).

To conclude, using modern mathematical models of memory, our results rigorously test suggestions of previous studies that age-related deficits are limited to the retrieval of specific, accurate, verbatim representations, sparing gist memory of meaning. They also indicate that mental representations become fuzzier during aging, which may allow older adults to have memory performance equivalent to that of younger adults in some cases, but also to make more semantic errors. 


\section{References}

Alghamdi, S. A., \& Rugg, M. D. (2020). The effect of age on recollection is not moderated by differential estimation methods. Memory, 28, 1067-1077.

Belacchi, C., \& Artuso, C. (2018). How taxonomic and thematic associations in semantic memory modulate recall in young through old-old adults. Psychology and Aging, 33, 1060-1069.

Brainerd, C. J., Gomes, C. F. A., \& Moran, R. (2014). The two recollections. Psychological Review, 121, 563-599.

Brainerd, C. J., Gomes, C. F. A., \& Nakamura, K. (2015). Dual recollection in episodic memory. Journal of Experimental Psychology: General, 144, 816-843.

Brainerd, C. J., \& Reyna, V. F. (2004). Fuzzy-trace theory and memory development. Developmental Review, 24, 396-439.

Brainerd, C. J., \& Reyna, V. F. (2010). Recollective and nonrecollective recall. Journal of Memory and Language, 63, 425-445.

Brainerd, C. J., \& Reyna, V. F. (2015). Fuzzy-trace theory and lifespan cognitive development. Developmental Review, 38, 89-121.

Brainerd, C. J., Reyna, V. F., \& Ceci, S. J. (2008). Developmental reversals in false memory: A review of data and theory. Psychological Bulletin, 134, 343-382.

Brainerd, C. J., Reyna, V. F., \& Howe, M. L. (2009). Trichotomous processes in early memory development, aging, and neurocognitive impairment: a unified theory. Psychological Review, 116, 783-832.

Brainerd, C. J., Reyna, V. F., \& Mojardin, A. H. (1999). Conjoint recognition. Psychological Review, 106, 160-179.

Brainerd, C. J., Reyna, V. F., Wright, R., \& Mojardin, A. H. (2003). Recollection rejection: false-memory editing in children and adults. Psychological Review, 110, 762-784. 
Brainerd, C. J., Wright, R., Reyna, V. F., \& Mojardin, A. H. (2001). Conjoint recognition and phantom recollection. Journal of Experimental Psychology: Learning, Memory, and Cognition, 27, 307-327.

Bugaiska, A., Clarys, D., Jarry, C., Taconnat, L., Tapia, G., Vanneste, S., \& Isingrini, M. (2007). The effect of aging in recollective experience: The processing speed and executive functioning hypothesis. Consciousness and Cognition, 16, 797-808.

Burke, S. N., Wallace, J. L., Nematollahi, S., Uprety, A. R., \& Barnes, C. A. (2010). Pattern separation deficits may contribute to age-associated recognition impairments. Behavioral Neuroscience, 124, 559-573.

Castel, A. D. (2005). Memory for grocery prices in younger and older adults: The role of schematic support. Psychology and aging, 20, 718-721.

Chen, T., \& Naveh-Benjamin, M. (2012). Assessing the associative deficit of older adults in long-term and short-term/working memory. Psychology and aging, 27, 666-682.

Davidson, P. S., \& Glisky, E. L. (2002). Neuropsychological correlates of recollection and familiarity in normal aging. Cognitive, Affective, \& Behavioral Neuroscience, 2(2), 174186.

Deese, J. (1959). On the prediction of occurrence of particular verbal intrusions in immediate recall. Journal of Experimental Psychology, 58, 17-22.

Devitt, A. L., \& Schacter, D. L. (2016). False memories with age: Neural and cognitive underpinnings. Neuropsychologia, 91, 346-359.

Flores, C. C., Hargis, M. B., McGillivray, S., Friedman, M. C., \& Castel, A. D. (2017). Gistbased memory for prices and "better buys" in younger and older adults. Memory, 25, 565573. 
Folstein, M. F., Folstein, S. E., \& McHugh, P. R. (1975). « Mini-mental state »: A practical method for grading the cognitive state of patients for the clinicians. Journal of Psychiatric Research, 12, 189-198.

Gallo, H. B., Hargis, M. B., \& Castel, A. D. (2019). Memory for weather information in younger and older adults: Tests of verbatim and gist memory. Experimental aging research, 45, 252-265.

Grady, C. (2012). The cognitive neuroscience of ageing. Nature Reviews Neuroscience, 13, 491-505.

Greene, N. R., \& Naveh-Benjamin, M. (2020). A specificity principle of memory: Evidence from aging and associative memory. Psychological Science, 31, 316-331.

Guillaume, F., Tison, C., \& Marzouki, Y. (2015). The interaction between memory trace and memory judgment in age-related decline. Experimental Aging Research, 41(5), 510-533.

Hay, J. F., \& Jacoby, L. L. (1999). Separating habit and recollection in young and older adults: Effects of elaborative processing and distinctiveness. Psychology and Aging, $14,122-134$.

Heck, D. W., Arnold, N. R., \& Arnold, D. (2018). TreeBUGS: An R package for hierarchical multinomial-processing-tree modeling. Behavioral Research Methods, 50, 264-284.

Hedden, T., \& Gabrieli, J. D. E. (2004). Insights into the ageing mind: A view from cognitive neuroscience. Nature Reviews Neuroscience, 5, 87-96.

JASP Team (2019). JASP (Version 0.9.2.0)[Computer software].

Jennings, J., \& Jacoby, L. L. (1993). Automatic versus intentional uses of memory: Aging, attention, and control. Psychology and Aging, 8, 283-293.

Kass, R. E., \& Raftery, A. E. (1995). Bayes factors. Journal of the American Statistical Association, 90, 773-795. 
Koen, J. D., \& Yonelinas, A. P. (2016). Recollection, not familiarity, decreases in healthy aging: Converging evidence from four estimation methods. Memory, 24, 75-88.

Koutstaal, W., \& Schacter, D. L. (1997). Gist-based false recognition of pictures in older and younger adults. Journal of Memory and Language, 37, 555-583.

Kruschke, J. K. (2011). Bayesian assessment of null values via parameter estimation and model comparison. Perspectives on Psychological Science, 6, 299-312.

Kruschke, J. K. (2018). Rejecting or accepting parameter values in Bayesian estimation. Advances in Methods and Practices in Psychological Science, 1, 270-280.

Langa, K. M., Plassman, B. L., Wallace, R. B., Herzog, A. R., Heeringa, S. G., Ofstedal, M. B., Burke, J. R., Fisher, G. G., Fultz, N. H., Hurd M. D., Potter G. G., Rodgers W. L., Steffens D. C., Weir D. R. \& Willis, R. J. (2005). The Aging, Demographics, and Memory Study: study design and methods. Neuroepidemiology, 25(4), 181-191.

Lavoie, D. J., \& Faulkner, K. (2000). Age differences in false recognition using a forced choice paradigm. Experimental Aging Research, 26, 367-381.

Naveh-Benjamin, M. (2000). Adult age differences in memory performance: tests of an associative deficit hypothesis. Journal of Experimental Psychology: Learning, Memory, and Cognition, 26, 1170-1187.

Naveh-Benjamin, M., Hussain, Z., Guez, J., \& Bar-On, M. (2003). Adult age differences in episodic memory: further support for an associative-deficit hypothesis. Journal of Experimental Psychology: Learning, Memory, and Cognition, 29, 826-837.

Parks, C. M., Murray, L. J., Elfman, K., \& Yonelinas, A. P. (2011). Variations in recollection: The effects of complexity on source recognition. Journal of Experimental Psychology: Learning, Memory, and Cognition, 37, 861-873.

Pettigrew, C., \& Martin, R. C. (2014). Cognitive declines in healthy aging: Evidence from multiple aspects of interference resolution. Psychology and Aging, 29, 187-204. 
R Core Team (2019). R: A language and environment for statistical computing. RStudio version 1.2.5033, (2019-12-04), [Computer Software]. Vienna, Austria. Retrieved from https://www.R-project.org/.

Reyna, V. F., \& Brainerd, C. J. (2011). Dual processes in decision making and developmental neuroscience: a fuzzy-trace model. Developmental Review, 31, 180-206.

Reyna, V. F., \& Mills, B. A. (2007). Interference processes in fuzzy-trace theory: Aging, Alzheimer's disease, and development. In D. S. Gorfein \& C. M. MacLeod (Eds.), Inhibition in cognition (p. 185-210).

Roediger, H. L., \& McDermott, K. B. (1995). Creating false memories: Remembering words not presented in lists. Journal of Experimental Psychology, 21, 803-814.

Rotello, C. M., Macmillan, N. A., \& Reeder, J. A. (2004). Sum-difference theory of remembering and knowing: a two-dimensional signal-detection model. Psychological Review, 111, 588-616

Rouder, J. N. (2014). Optional stopping: No problem for Bayesians. Psychonomic bulletin \& review, 21, 301-308.

Rouder, J. N., Morey, R. D., Speckman, P. L., \& Province, J. M. (2012). Default Bayes factors for ANOVA designs. Journal of Mathematical Psychology, 56, 356-374.

Smith, J. B., \& Batchelder, W. H. (2010). Beta-MPT: Multinomial processing tree models for addressing individual differences. Journal of Mathematical Psychology, 54, 167-183.

Stahl, C., \& Klauer, K. C. (2008). A simplified conjoint recognition paradigm for the measurement of gist and verbatim memory. Journal of Experimental Psychology: Learning, Memory, and Cognition, 34, 570-586.

Tinard, S., \& Guillaume, F. (2019). Age-related differences in the impact of prior knowledge on recognition performance: A face recognition study. Experimental Aging Research, 45(2), 154-166. 
Tun, P. A., Wingfield, A., Rosen, M. J., \& Blanchard, L. (1998). Response latencies for false memories: Gist-based processes in normal aging. Psychology and Aging, 13, 230-241. underpinnings. Neuropsychologia, 91, 346-359.

West, R. L. (1996). An application of prefrontal cortex function theory to cognitive aging. Psychological bulletin, 120, 272-292.

Wixted, J. T., \& Mickes, L. (2010). A continuous dual-process model of remember/know judgments. Psychological Review, 117, 1025-1054.

Yonelinas, A. P. (2002). The nature of recollection and familiarity: A review of 30 years of research. Journal of memory and language, 46, 441-517.

Zacks, R. T., \& Hasher, L. (2006). Aging and long-term memory: Deficits are not inevitable. In E. Bialystok \& F. I. M. Craik (Eds.), Lifespan cognition: Mechanism of change (pp. 162-177). New York, NY: Oxford University Press. 